\title{
Designing a Low- Pass FIR Digital Filter by using Rectangular, Hanning and Triangular Window Technique
}

\author{
Kamal Kant Chandra ${ }^{1}$, Mohan Lal prajapati ${ }^{2}$, Mr. Pranay Kumar Rahi ${ }^{3}$ \\ BE Scholar, Dept of Electrical \& Electronics Engineering, Institute of Technology Korba, Chhattisgarh, India ${ }^{1,2}$
}

Assistant Professor, Dept of Electrical \& Electronics Engineering, Institute of Technology Korba, Chhattisgarh, India ${ }^{3}$

\begin{abstract}
Digital filter are widely used in the world of communication and computation. On the other hand, to design a Digital Finite Impulse Response (FIR) filter that satisfies all the required condition is challenging one. In this paper, design techniques of Low Pass filter using Rectangular, Hanning and Triangular Window Techniques of order (15) are presented. It shown that filter design by using Rectangular Window Technique is better as it provides better result in terms of magnitude, phase, impulse, step responses and pole-zero plot. The analysis of magnitude, phase, impulse, step response and pole-zero plot of proposed FIR Low Pass filter is performed using MATLAB Simulation.
\end{abstract}

Keywords: DSP, Digital filter, Low-Pass filter, FIR filter, Rectangular window, Hanning and Triangular Window Techniques.

\section{INTRODUCTION}

A digital filter is a system which passes some desired signals more than others to reduce or enhance certain aspects of that signal. It can be used to pass the signals according to the specified frequency pass-band and reject the other frequency than the pass-band specification.[1] In the FIR system, the impulse response is of finite duration, this means that it has a finite number of nonzero terms. On the other hand, The IIR system has an infinite number of nonzero terms. This means its impulse response is of infinite duration. While implementation, FIR filter needs no feedback. FIR filter is not a recursive filter. Because of this reason, the structure of FIR filter is much more simpler than compared to the IIR filter [2] The structure of these algorithms uses a repetitive delay-andadd format that can be represented as "Direct Form-I Structure". The advantage of FIR filter over IIR filters are, FIR filter has linear phase andeasily to control where as IIR filter has no particular phase and difficult to control. FIR filter is stable and depends only on input.[3] Digital filters have characteristics such as linear phase response. If we compare digital filter with analog.[4] Filter they are preferred in number of application like speech processing, image processing and data compression [5]

It is calculated by applying an impulse signal at the input. The values of $h(n)$ will determine the type of the filter e.g. low pass, high pass, band pass etc. the values of $h(n)$ are to be determine in the design process and $\mathrm{N}$ represents the order of the polynomial function.[6]

\subsection{RECTANGULAR WINDOW FUNCTION}

The rectangular window (sometimes known as the0T 0T32Tboxcar0T32T 0Tor0T 0T32TDirichlet(0T32T
0Twindow) is the simplest window, equivalent to replacing all but0T 0TNOT 0Tvalues of a data sequence by zeros, making it appear as though the waveform suddenly turns on and off. The weighting function for the rectangular window is given by

$$
\mathrm{WR}(\mathrm{n})=\left\{\begin{array}{c}
1 \text { for }|\mathrm{n}| \leq \frac{\mathrm{M}-1}{2} \\
0, \text { otherwise } \ldots \ldots \ldots \ldots \ldots \ldots \ldots \ldots
\end{array}\right.
$$

\section{SIMULATION AND RESULT}

Table 1: Parameter Specification

\begin{tabular}{|l|l|}
\hline PARAMETER & VALUES \\
\hline Sampling Frequency(Fs) & $48000 \mathrm{~Hz}$ \\
\hline Cut off Frequency $(\mathrm{Fc})$ & $10800 \mathrm{~Hz}$ \\
\hline Order(N) & 15 \\
\hline
\end{tabular}

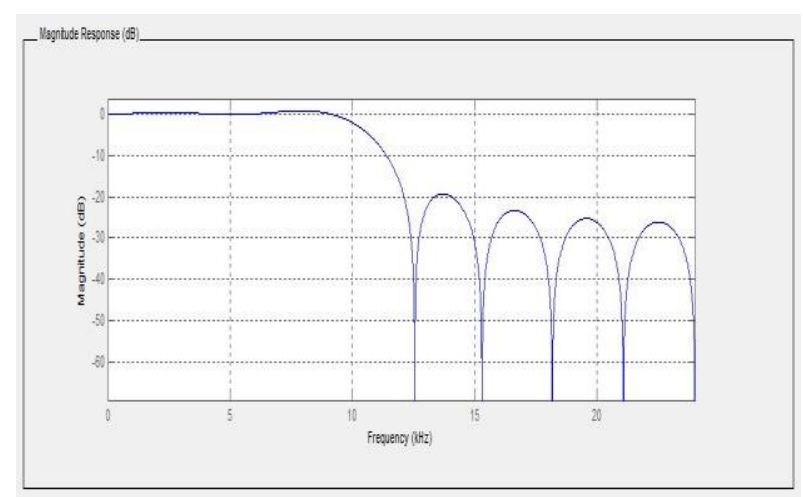

Fig 1: Magnitude response of Rectangular Window Technique 


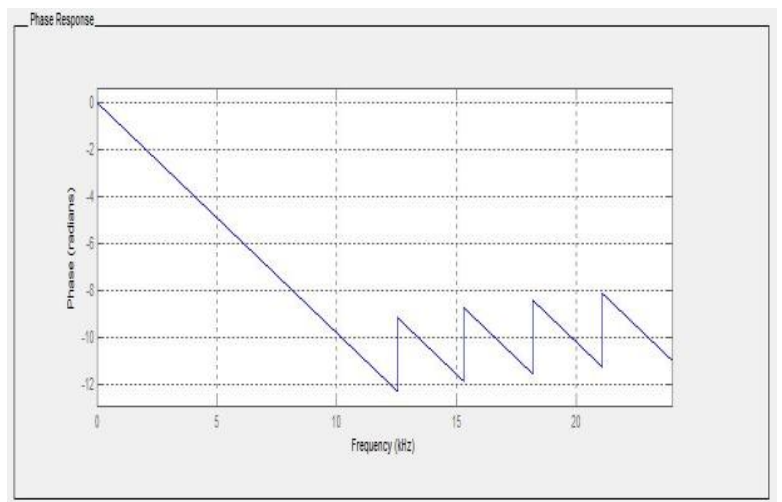

Fig2: Phase response of Rectangular Window Technique

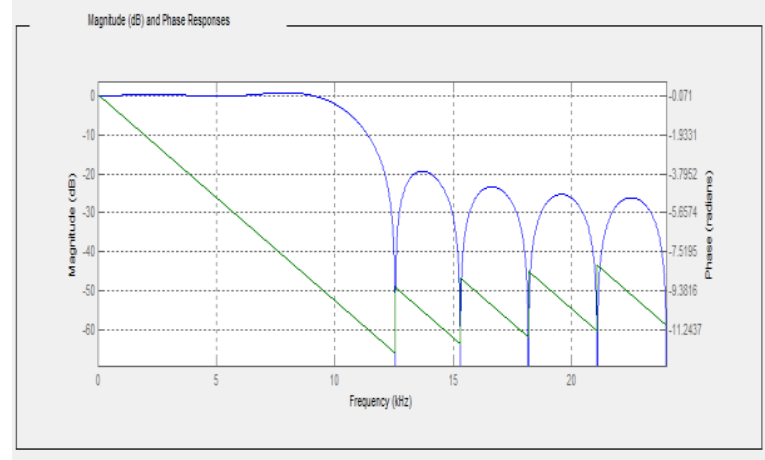

Fig 3: Magnitude and Phase Response rectangular window technique

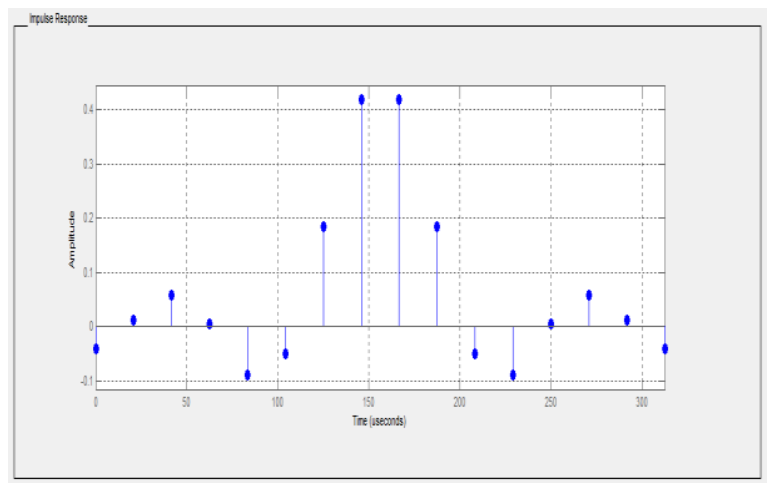

Fig 4: Impulse Response of rectangular Window technique

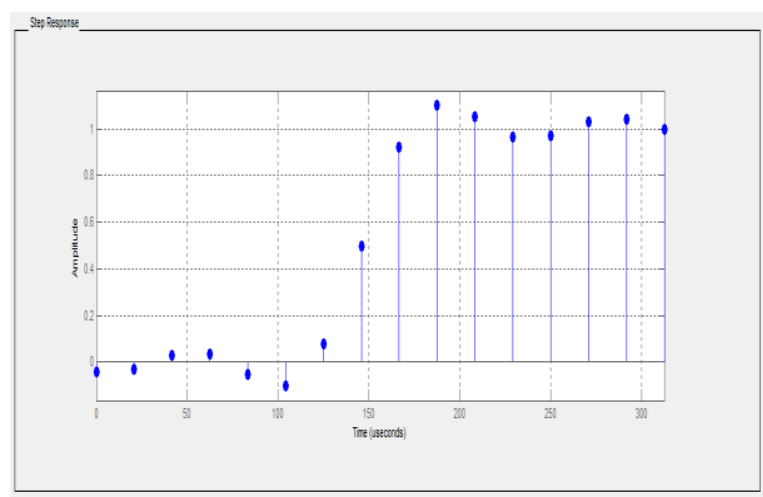

Fig5: Step Response of rectangular window Technique

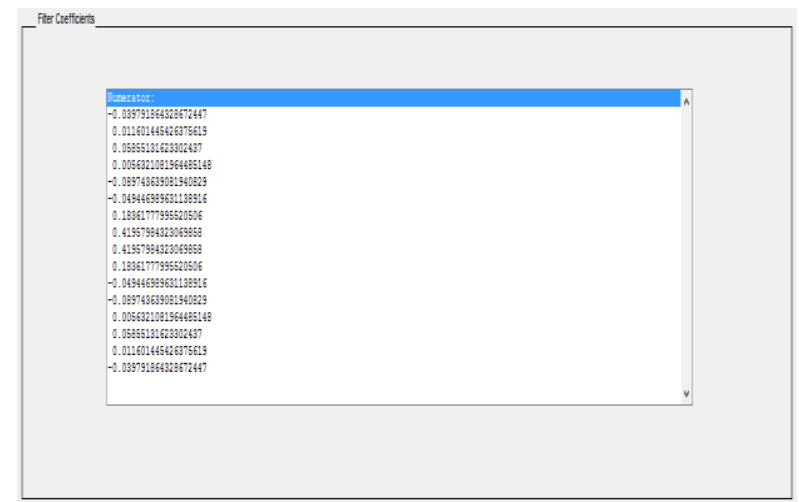

Fig6: Pole/Zero plot of Rectangular Window Technique

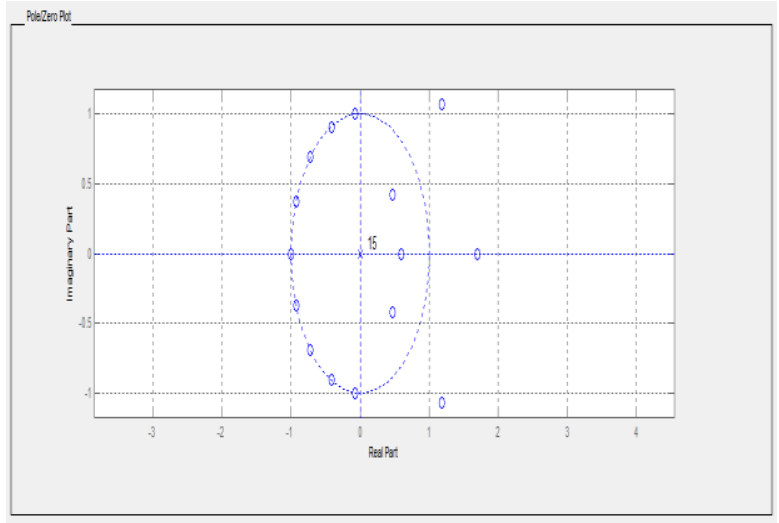

Fig 7: Filter coefficient of Rectangular Window Technique

\subsection{HANNING WINDOW FUNCTION}

The Hann window named after Julius von Hann and also known as the Hanning (for being similar in name and form to the Hamming window), von Hann and the raised cosine window is defined by (with hav for the haversine function):

$$
\mathrm{W}(\mathrm{n})=0.5 \quad\left(1-\cos \left(\frac{2 \pi}{\mathrm{N}-1}\right)\right)=\operatorname{Hav}\left(\frac{2 \pi \mathrm{n}}{\mathrm{N}-1}\right)
$$

The ends of the cosine just touch zero, so the side-lobes roll off at about $18 \mathrm{~dB}$ per octave.

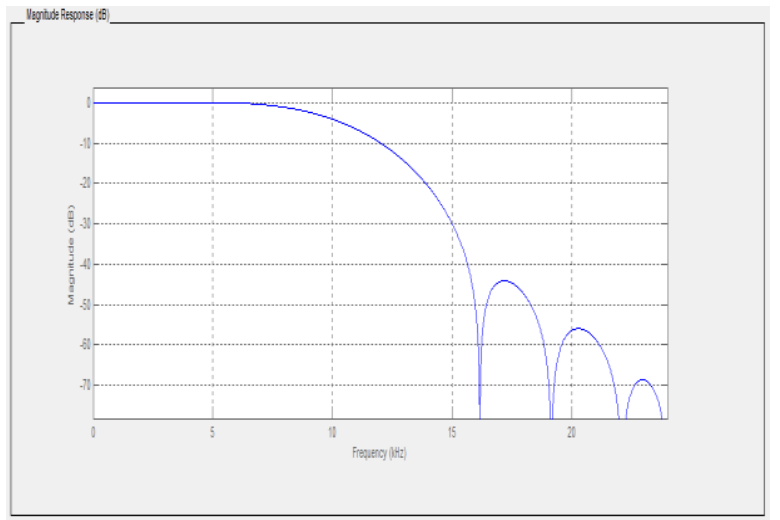

Fig 8: Magnitude response of Hanning Window Technique 


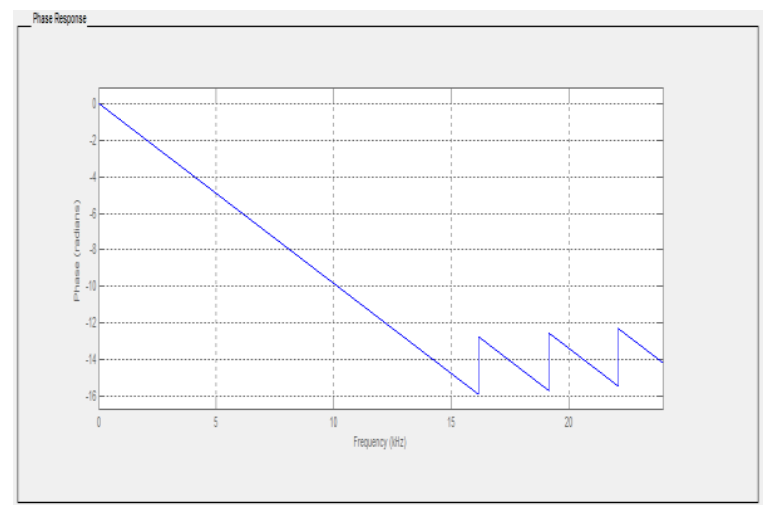

Fig 9: Phase response of Hanning Window Technique

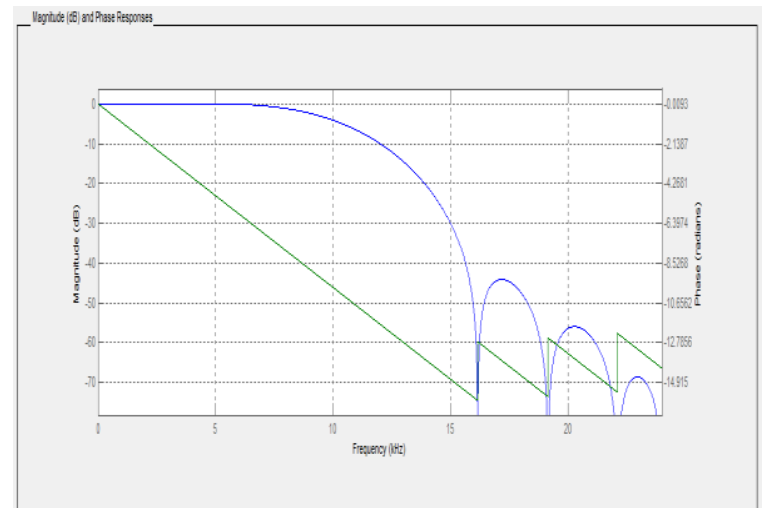

Fig 10: Magnitude and Phase Response Hanning window technique

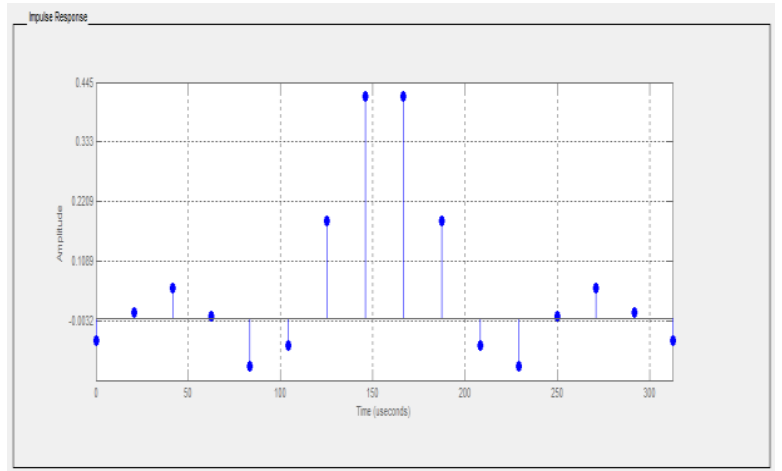

Fig 11: Impulse Response of hanning Window technique

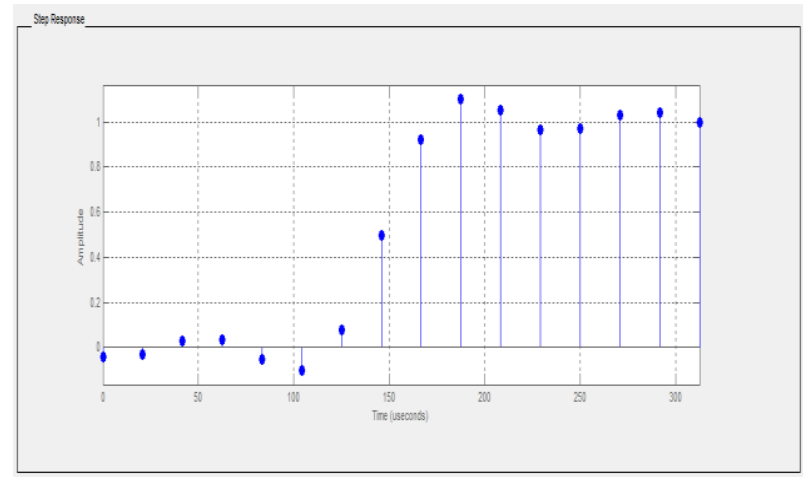

Fig 12: Step Response of hanning window Technique

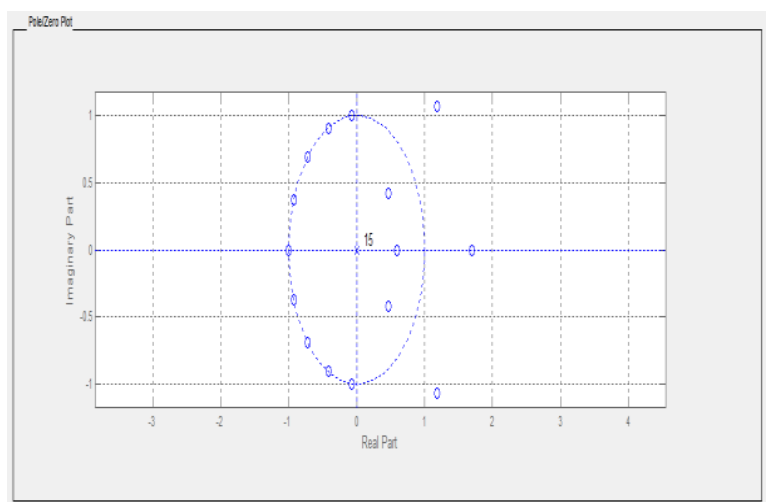

Fig13: Pole/Zero plot of hanning Window Technique

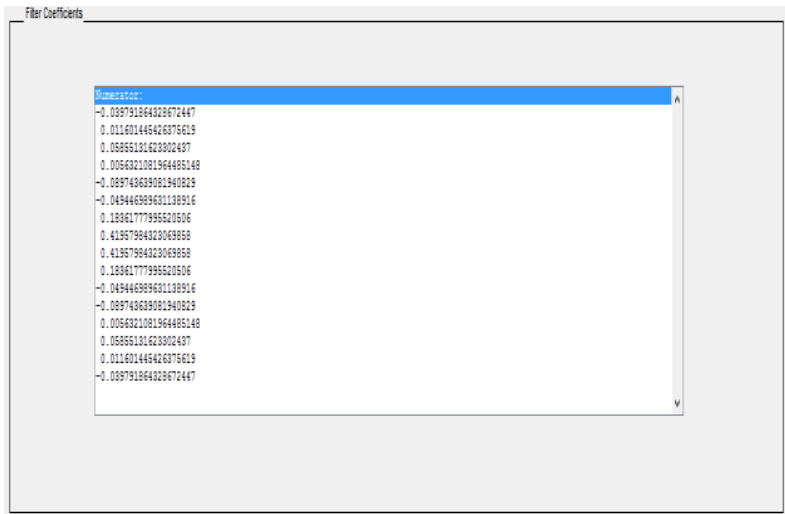

Fig 14: Filter coefficient of Rectangular Window Technique

\subsection{TRIANGULAR WINDOW FUNCTION}

The main problem associated with he rectangular Window is the presence of ripple near the band edges of the resulting filter,which are caused by the existence Of sidelobes in the frequency response of the window. Such a problemis due to the inherent discontinuty is to the rectangular window in the time domain. One way to reduce such a discontinuity is to employ a triangular shaped window, which will present only small it discontinuities near its edges.

The standard triangular window is define as

$$
\mathrm{w}_{\mathrm{t}}(\mathrm{n})=\left\{\frac{2|\mathrm{n}|}{\mathrm{M}}+1, \text { for }|\mathrm{n}| \leq \frac{\mathrm{M}}{2}\right\}
$$

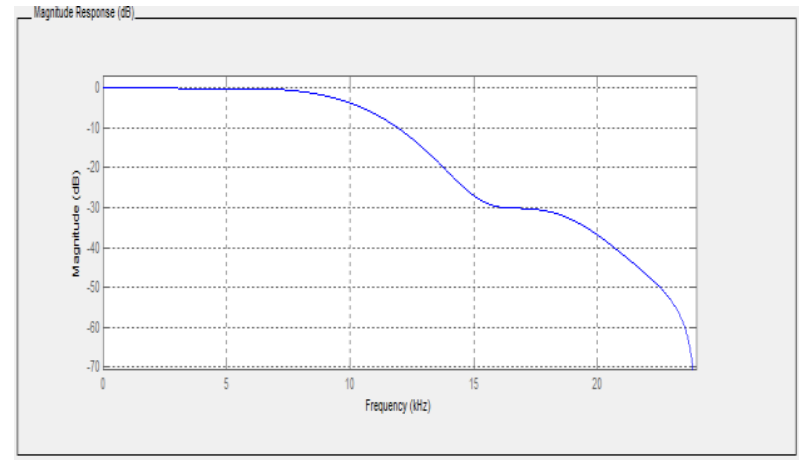

Fig15: Magnitude response of Triangular Window Technique 


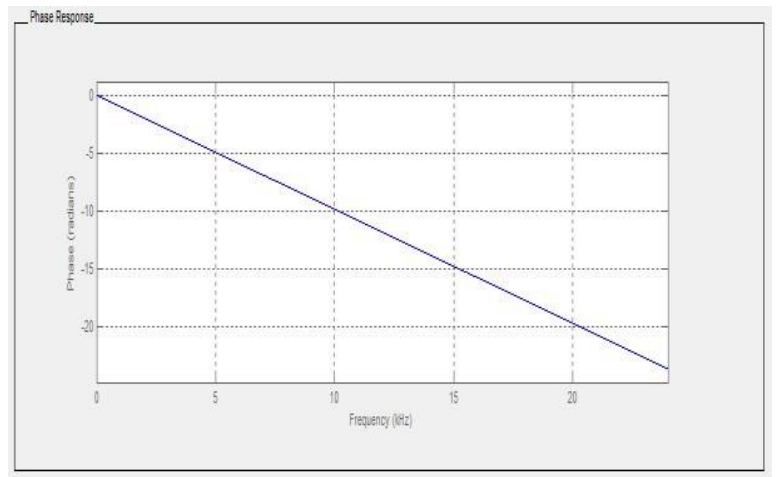

Fig 16: Phase response of Triangular Window Technique

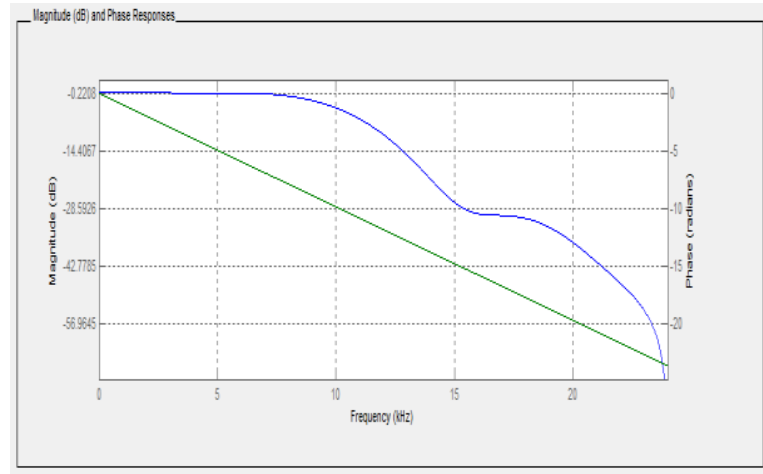

Fig 17: Magnitude and Phase Response of Triangular window technique

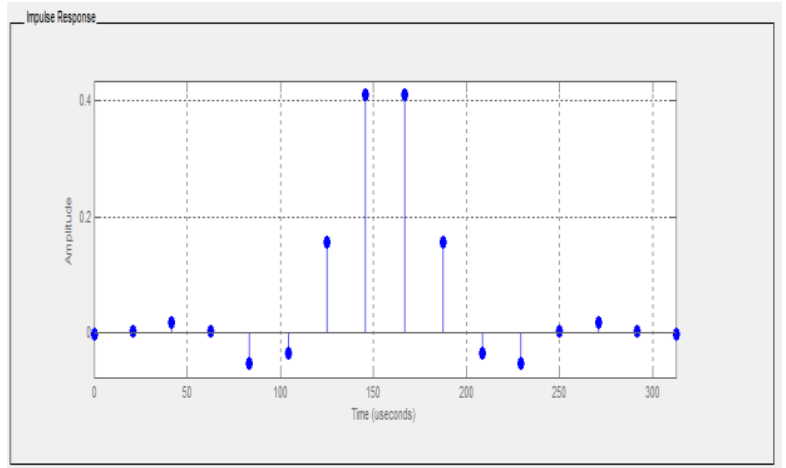

Fig 18: Impulse Response of Triangular Window technique

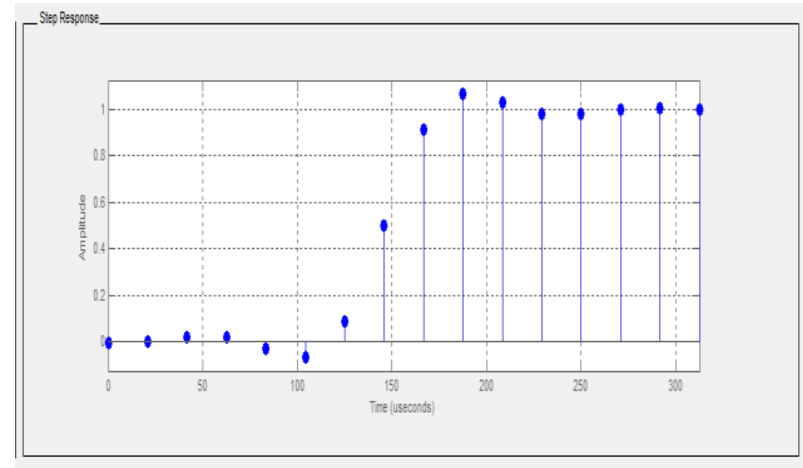

Fig 19: Step Response of Triangular window Technique

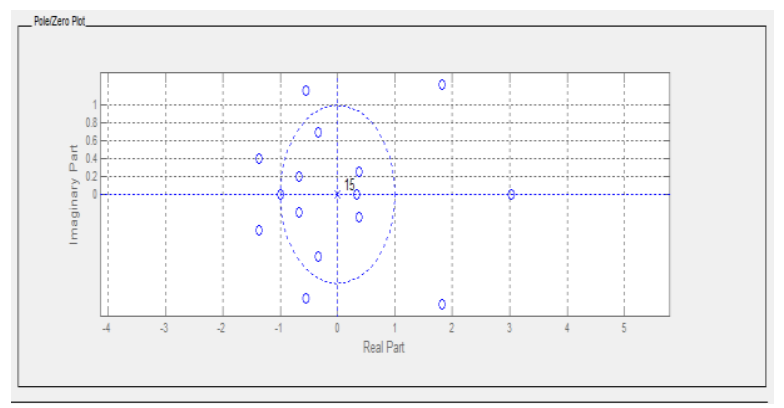

Fig20: Pole/Zero plot of Triangular Window Technique

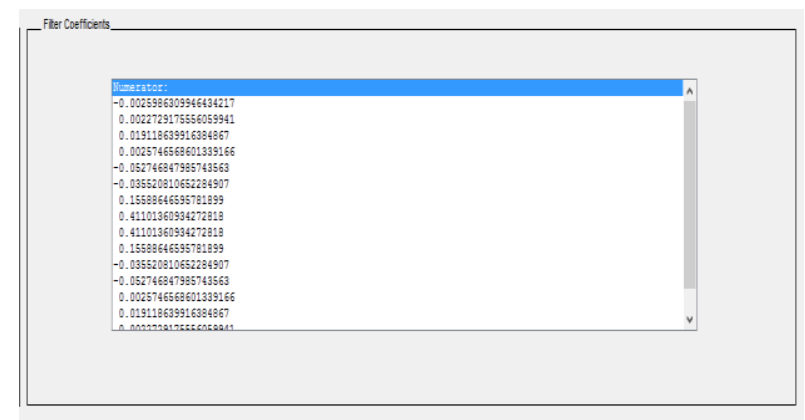

Fig 21: Filter coefficient of Triangular Window Technique

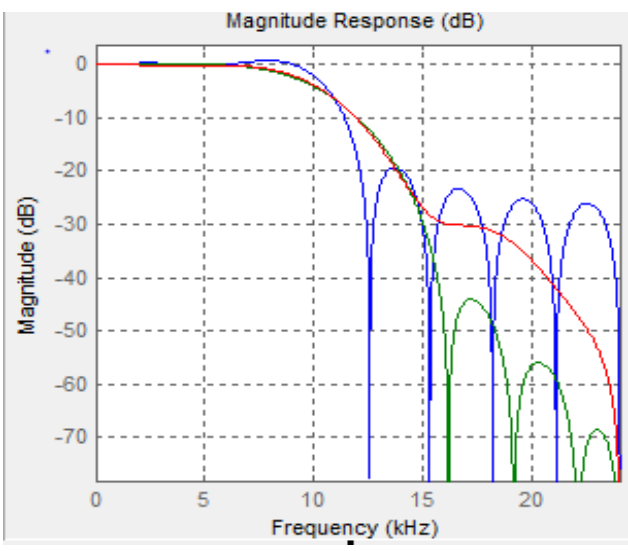

Fig 22: Magnitude comparison of rectangular, hanning And triangular window

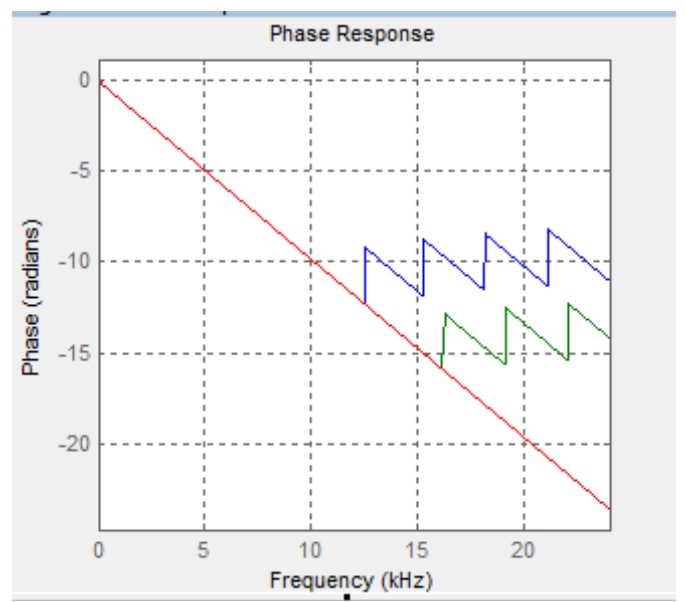

Fig 23: Phase comparison of rectangular, hanning And triangular window 


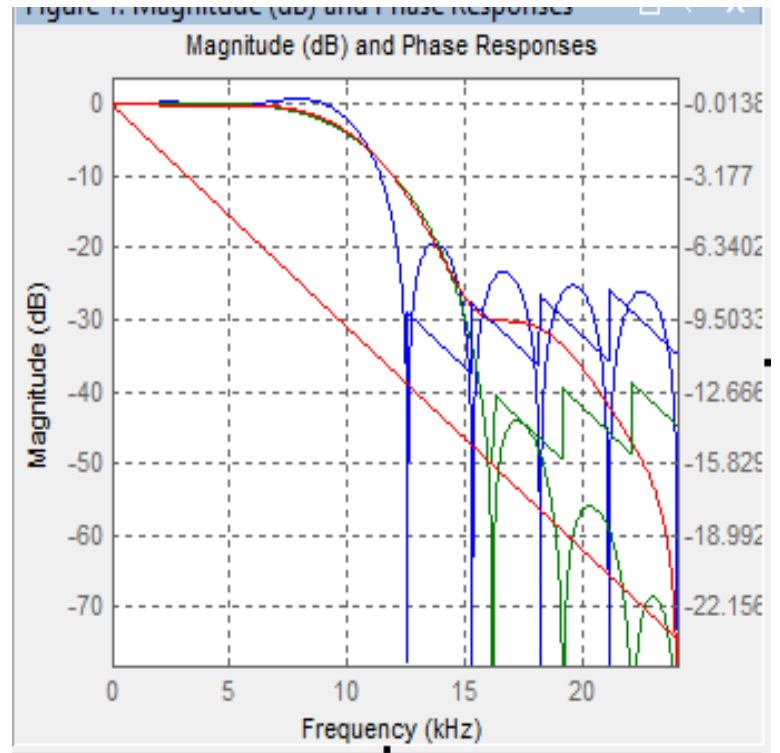

Fig 24: Magnitude and phase comparison rectangular, hanning and triangular window

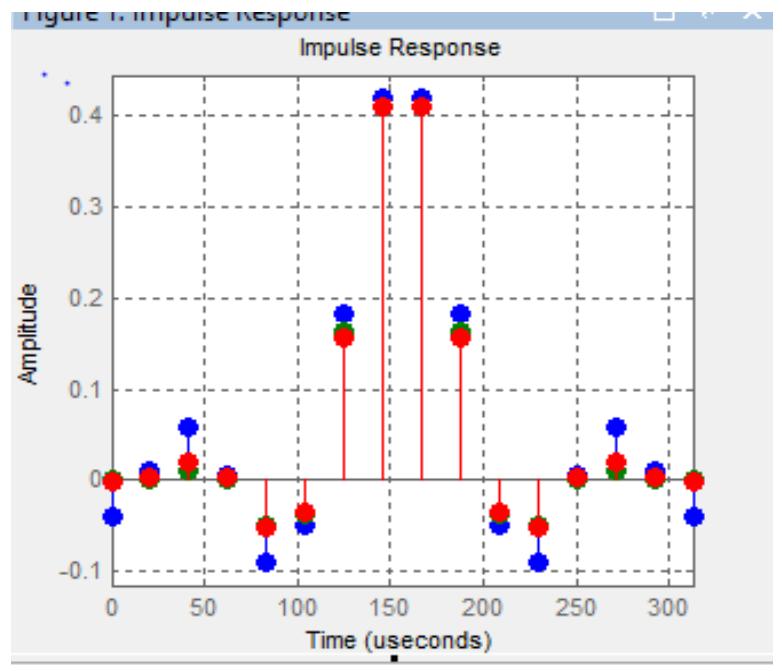

Fig25: Impulse comparsion of rectangular, hanning and triangular window

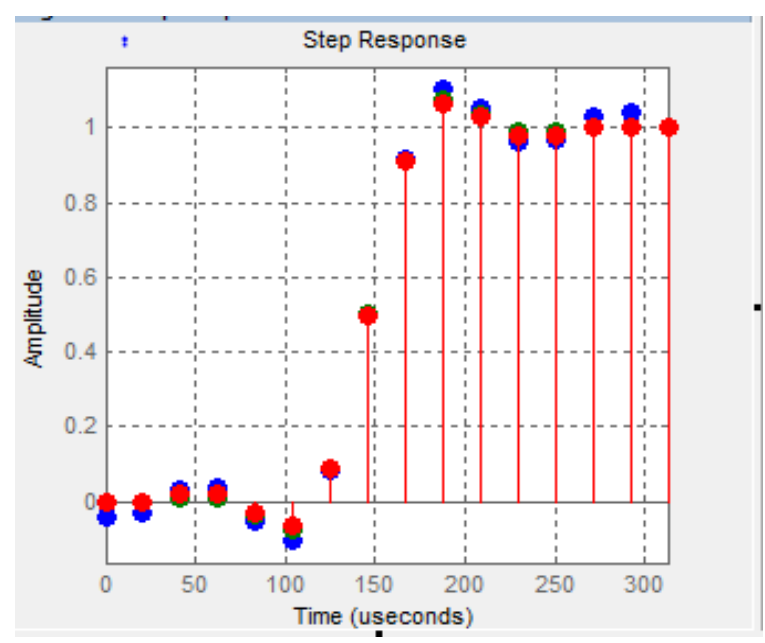

Fig.26: step comparison of rectangular, hanning and triangular

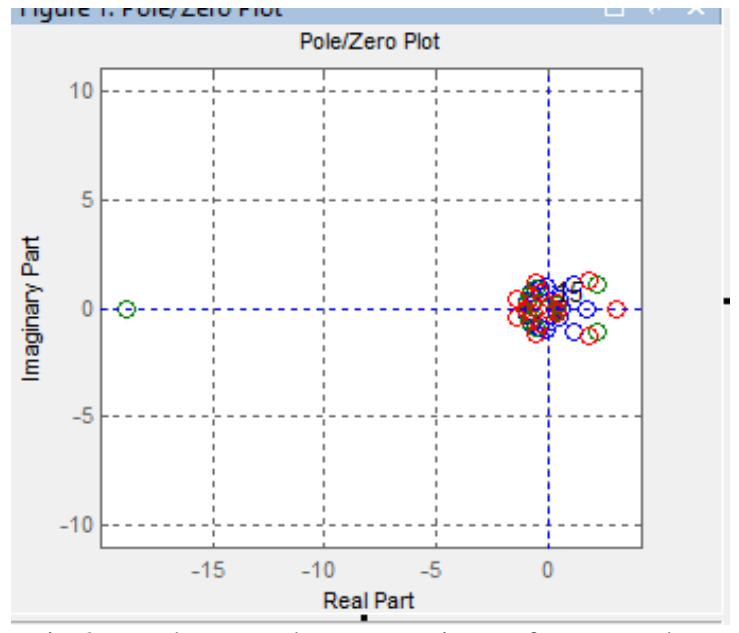

Fig 27: Pole zero plot comparison of rectangular, Hannning and triangular

TABLE 2 Frequency and magnitude

\begin{tabular}{|c|c|c|c|}
\hline Frequency & Rectangular & Hanning & Triangular \\
\hline $0.1 \pi$ & 0.136 & 0.002 & -0.028 \\
\hline $0.2 \pi$ & 0.282 & 0.014 & -0.095 \\
\hline $0.3 \pi$ & 0.220 & 0.043 & -0.143 \\
\hline $0.4 \pi$ & -0.028 & 0.067 & -0.155 \\
\hline $0.5 \pi$ & -0.160 & 0.030 & -0.143 \\
\hline $0.6 \pi$ & 0.312 & -0.136 & -0.186 \\
\hline $0.7 \pi$ & 0.618 & -0.521 & -0.431 \\
\hline $0.8 \pi$ & 0.710 & -1.511 & -1.603 \\
\hline $0.9 \pi$ & -0.395 & -2.610 & -2.133 \\
\hline$\Pi$ & -2.605 & -4.383 & -4.769 \\
\hline
\end{tabular}

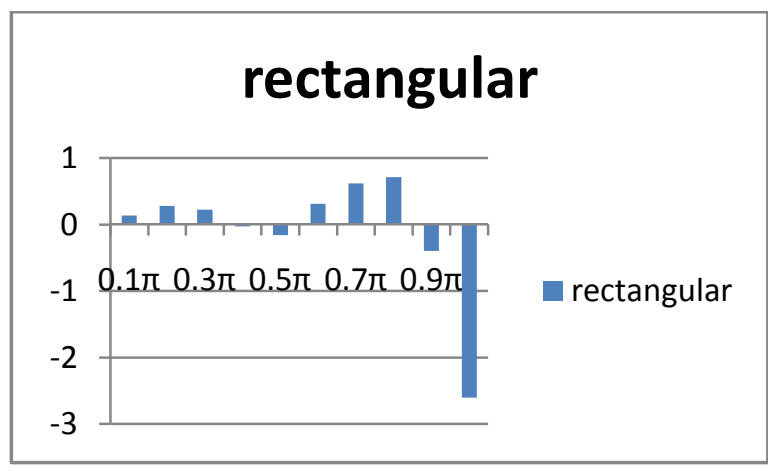

Fig28: Frequency and magnitude plot of rectangular window

\section{Hanning}

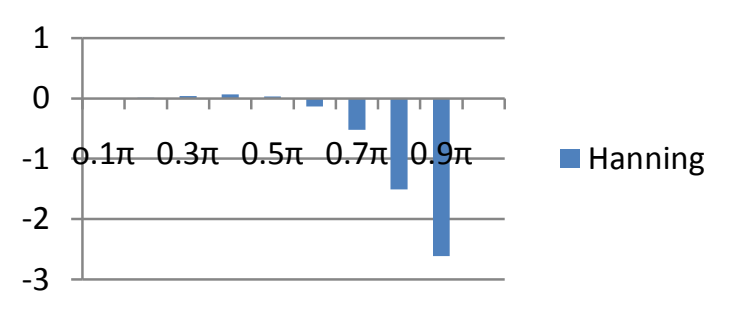

Fig 29: Frequency and magnitude plot of hanning window 


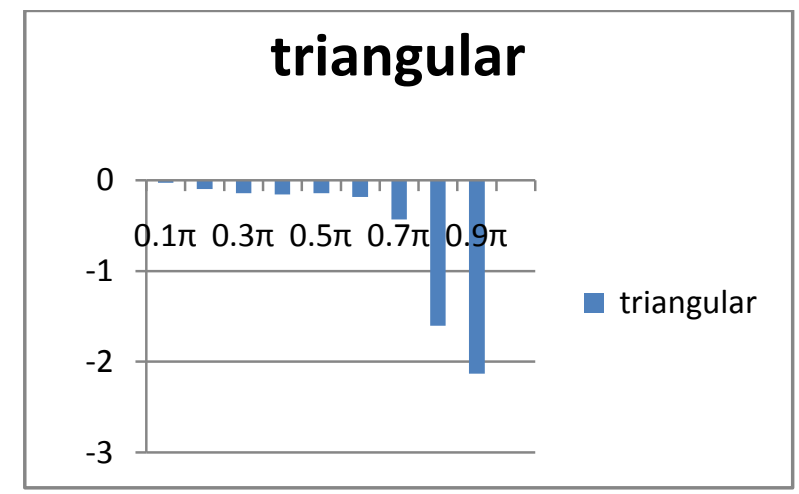

Fig 30: Frequency and magnitude plot of triangular window

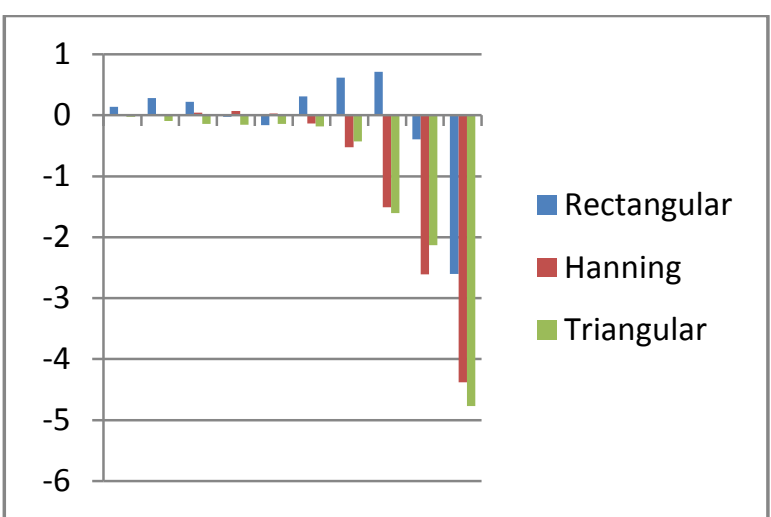

Fig31: Magnitude and frequency plot of rectangular, hanning and triangular

\section{CONCLUSION}

In this research paper Low pass FIR filter has been designed using MATLAB Rectangular, Hanning and triangular window technique. It concludes by comparative values of both magnitude and phase response of the filter using both the techniques at same frequency i.e. $\mathrm{fRsR}=45000 \mathrm{~Hz}$ and $\mathrm{fRcR}=10800 \mathrm{~Hz}$. present with the wanted signals too.

\section{FILTER SPECIFICATION}

Before a filter can be design, a set of filter specification must be define, For e.g suppose that we would like to design a low pass filter with a cut of frequency $\mathrm{w}_{\mathrm{c}}$. The frequency response of an ideal low pass filter with linear phase and a cut of frequency $\mathrm{w}_{\mathrm{c}}$ is

$$
\mathrm{H}_{\mathrm{d}}\left(\mathrm{e}^{\mathrm{jw}}\right)= \begin{cases}e^{-j w} & |\boldsymbol{w}| \leq \boldsymbol{w c} \\ 0 & \boldsymbol{w} \boldsymbol{c} \leq|\boldsymbol{w}| \leq \pi\end{cases}
$$

Which has a unit sample response

$$
\mathbf{h}_{\mathbf{d}}(n)=\frac{\sin (n-a) w c}{\pi(n-a)}
$$

Because this filter is unrealizable (non causal and unstable) it is necessary to relax the ideal constraints on the frequency response and allow some deviation from the ideal response. The specification for a low pass filter will typically have the form.

\section{REFERENCES}

[1] Sandeep Kaur, Sangeet Pal Kaur, "Design of FIR filter using hanning window, hamming window and modified hamming window", Vol 4 Issue 5, May 2015.

[2] M.Mukta Dir Rahman, "Comparative Performance Analysis of Hamming, hanning and Blackman Window" Vol 96- No.18, June 2014.

[3] S Salivahanan, C Gnanapriya, A Vallavaraj "Digital signal processing "McGraw-Hill Education, $2^{\text {nd }}$ Edition.

[4] Ankan Bhattacharya, "A modified window function for FIR filter design with an improved frequency response and its comparison with the Hamming window" International Journal of Science, Engineering and Technology Research (IJSETR) Vol 2, Issue 5, May 2013.

[5] Manjinder Kaur, Sangeet Pal Kaur, “ FIR Low Pass Filter Designing Using Different Window Functions and their Comparison using MATLAB" (An ISO 3297: 2007 Certified Organization) Vol 4 Issue 5, May 2015.

[6] U.A.Bakshi, Dr. J.S. Chitode, "Signals and Systems" Technical Publications.

\section{BIOGRAPHIES}

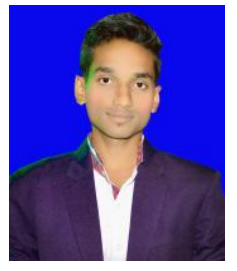

Kamal Kant Chandra pursuing Bachelor of Engineering in Electrical and Electronics Engineering in $6^{\text {th }}$ semester from Institute of Technology Korba, Chhattisgarh Swami Vivekananda Technical University, Chhattisgarh, India

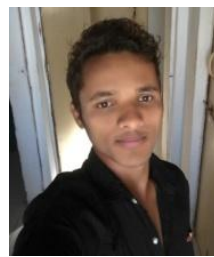

Mohan Lal Prajapati pursuing Bachelor of Engineering in Electrical and Electronics Engineering in $6^{\text {th }}$ semester from Institute of Technology Korba Chhattisgarh Swami Vivekananda Technical University Chhattisgarh, India

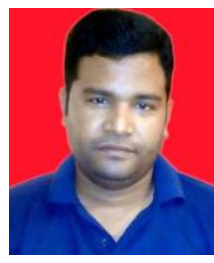

Pranay Kumar Rahi Received the Bachelors of Engineering degree in Electronics and Telecommunication Engineering from Government Engineering College, Guru Ghasidas University, Bilaspur, Chhattisgarh, India in 2004, working as a Assistant Professor in Electrical and Electronic Engineering of Institute of Technology Korba since 2008 and pursuing Masters of Engineering in Electronics and Communication Engineering from National Institute of Technical Teacher's Training\& Research, Punjab University, Chandigarh, India. Presently working as assistant professor in Department of Electrical and Electronic Engineering, Institute of Technology Korba, Chhattishgarh since 2008. He has authored More than 40 research publications and published journal papers and research papers in the leading International and National journal. His primary research interest includes Digital Signal Processing, VLSI Design, Control Systems and Digital Electronics and logic design. 